\title{
Microdialysis of the spinal cord during thoracic aortic cross-clamping in a porcine model
}

\author{
OD Sæther*,1, T Bäckström², P Aadahl ${ }^{3}$, HO Myhre ${ }^{1}$, L Norgren ${ }^{1}$ and U Ungerstedt ${ }^{2}$ \\ ${ }^{1}$ Department of Surgery, Trondheim University Hospital, Norway; ${ }^{2}$ Department of Physiology and Pharmacology, \\ Karolinska Institute, Stockholm, Sweden; ${ }^{3}$ Department of Anaesthesiology, Trondheim University Hospital, Norway
}

\begin{abstract}
Objective: Utilising microdialysis to measure the changes of glucose, lactate, pyruvate and glutamate levels in the spinal cord after cross-clamping of the thoracic aorta in an established porcine model to monitor the degree of ischaemia.
\end{abstract}

Design: Experimental study with a porcine model.

Setting: University Hospital, Trondheim.

Subjects: Six pigs.

Main outcome measure: Lactate, pyruvate and glutamate concentrations in the microdialysis perfusate from the spinal cord.

Results: A significant increase of the lactate-pyruvate ratio during the last $30 \mathrm{~min}$ of the $1 \mathrm{~h}$ clamping period, with a maximum increase of $169 \%$ from the basal value the last $10 \mathrm{~min}$ before declamping. No evident change in this ratio between the clamping and the reperfusion period. No significant change in glutamate levels during clamping or reperfusion period.

Conclusion: Microdialysis reflects the ischaemic state of the spinal cord during crossclamping of the thoracic aorta in pigs, and is well suited to study each phenomena.

Spinal Cord (2000) 38, 153-157

Keywords: aortic cross-clamping; spinal cord ischaemia; microdialysis; pig

\section{Introduction}

During thoracoabdominal aortic aneurysm surgery, paraplegia occurs in $5-30 \%{ }^{1}$ The aetiology of spinal cord injury is multifactorial, and the main reasons are decreased perfusion pressure during surgery, failure to re-establish blood flow to the spinal cord after the repair and a biochemically mediated reperfusion injury. Reduction of cerebrospinal fluid pressure (CSFP), hypothermia and pharmacological protection of the spinal cord have been tried for prevention as have various shunting and perfusion procedures. ${ }^{2}$ The metabolism of the spinal cord during proximal aortic cross-clamping are not entirely clear and it is therefore of interest to study ischaemic effects on the spinal cord.

In a porcine experimental model, previously described by us, thoracic aortic cross-clamping caused a significant decrease of laser-Doppler flux (LDF), measured in the spinal cord at the L2-L4 level, to about $40 \%$ of baseline values. Complementary occlusion of the azygos vein decreased the perfusion even more (to $17 \%$ of baseline LDF values). ${ }^{3}$

Microdialysis is a technique for sampling small quantities of fluid from the individual tissues with a thin dialysis tube perfused with a physiological liquid. Diffusion of substances back and forth over the

*Correspondence: OD Sæther, Department of Surgery, Trondheim University Hospital, 7006 Trondheim, Norway dialysis membrane gives a dialysis perfusate reflecting the composition of the extracellular fluid. ${ }^{4}$ Microdialysis has frequently been used to study brain ischaemia and brain injuries. ${ }^{4-6}$ In animal studies a certain increase of the excitatory amino acid glutamate level is recorded. ${ }^{7-10}$ During brain and spinal cord ischaemia a glutamate increase has been proposed as a possible risk factor for irreversible neuronal damage. ${ }^{11,12}$ Microdialysis of the spinal cord in larger animals has to our knowledge only been described by one research group. ${ }^{12,13}$

The purpose of the present study was to use our porcine model in a pilot study to evaluate the effect on microdialysis parameters (glucose, lactate, pyruvate and glutamate) from the spinal cord following crossclamping of the thoracic aorta including azygos vein occlusion, to monitor the degree of spinal cord ischaemia.

\section{Materials and methods}

Anaesthesia and experimental conditions

Six Norwegian land-race pigs with a median weight of $23 \mathrm{~kg}$ were premedicated with azaperone $(15 \mathrm{mg} /$ $\mathrm{kg}$ i.m.) and diazepam ( $1 \mathrm{mg} / \mathrm{kg}$ i.m.). Anaesthesia was induced with injections of pentobarbital sodium 
$(25 \mathrm{mg} / \mathrm{kg})$, ketamine $(20 \mathrm{mg} / \mathrm{kg})$ and atropine $(1 \mathrm{mg})$ through an ear vein, and was then maintained with ketamine $(20 \mathrm{mg} / \mathrm{kg} / \mathrm{h}$ i.v. $)$, fentanyl $(0.05 \mathrm{mg} / \mathrm{kg} / \mathrm{h}$ i.v. $)$ and pancuronium bromide $(0.14 \mathrm{mg} / \mathrm{kg} / \mathrm{h}$ i.v. $)$.

The pigs were tracheostomized and ventilated with $40 \% \mathrm{O}_{2}$ in room air by a dual phase respirator pump (Harvard Apparatus, USA) with a respiration rate of 12 strokes per minute and tidal volume of 350 . A solution of glucose $(35 \mathrm{mg} / \mathrm{ml})$ and $\mathrm{NaCl}(3 \mathrm{mg} / \mathrm{ml})$ was infused $(10 \mathrm{ml} / \mathrm{kg} / \mathrm{h}$ i.v. $)$ for basal fluid requirements. A catheter was inserted into the right femoral vein for additional volume infusion at declamping of the aorta. During the experiment a heating pad maintained body temperature at $37^{\circ} \mathrm{C}$.

A left thoracotomy was performed in the fifth interspace. The descending aorta as well as the azygos vein were dissected, and the aorta was prepared for cross-clamping just distal to the left subclavian artery.

A two-segment laminectomy was performed at the L2 - L3 level. After skin incision, the muscles attached to the spinous processes and laminae of the L2 and L3 vertebrate were separated from bone by blunt dissection. The spinous processes of L2 and L3 were removed with a bone cutting forceps, and the laminae excised with a rongeur. After laminectomy, the epidural fat was gently dissected away to expose the dura mater. The dura and the arachnoid membrane were opened with a longitudinal incision in the midline over the entire length of the laminectomy.

A CMA-12 microdialysis probe (CMA Microdialysis AB; Stockholm, Sweden) with a membrane length of $2 \mathrm{~mm}$ was then inserted into the spinal cord in a ventrolateral direction as to be located in the grey matter.

After a stabilisation period of $60 \mathrm{~min}$, collection of microdialysis samples was started. Basal microdialysate levels were obtained as one sample during $30 \mathrm{~min}$, after which the thoracic aorta was cross-clamped and the azygos vein occluded for $1 \mathrm{~h}$. The clamps were then removed and further samples were collected for $30 \mathrm{~min}$. During clamping and in the reperfusion period dialysis sampling was done in 10 -min fractions.

During cross-clamping $16 \mu \mathrm{g} / \mathrm{kg} / \mathrm{min}$ sodium nitroprusside and $50 \mathrm{~mm}$ of sodium bicarbonate were infused to control hypertension and to prevent acidosis respectively. Five minutes before declamping sodium nitroprusside was stopped, the respiration rate was increased to $20 / \mathrm{min}$ and $500 \mathrm{ml}$ of $3.5 \%$ gelatine solution (Haemaccel ${ }^{\circledR}$ ) was infused i.v. After declamping no vasoactive medication was given.

At the conclusion of each experiment, the animals were sacrificed with an overdose of pentobarbital sodium i.v. In two cases, the spinal cord was collected and examined with standard histopathological techniques in order to identify the exact placement of the microdialysis probe within the spinal cord.

\section{Microdialysis}

The perfusion fluid was a Ringer solution containing $147 \mathrm{mM} \mathrm{Na}^{+}, 4 \mathrm{mM} \mathrm{K}^{+}, 2.3 \mathrm{mM} \mathrm{Ca}^{2+}$, and $155.6 \mathrm{mM}$
$\mathrm{Cl}$. The probes were connected to a microinfusion pump (CMA 102: CMA Microdialysis AB) and perfused at a rate of $2 \mu \mathrm{l} / \mathrm{min}$.

\section{Chemical analysis}

The samples were frozen to $-20^{\circ} \mathrm{C}$ overnight, packed in dry ice, and transported to the analysis laboratory.

For the lactate and pyruvate analysis, 5-10 $\mu$ of the sample were injected with a CMA 200 autoinjector onto an HPLC system equipped with a polymericresin-based column (polypore $\mathrm{H}$. $10 \mu \mathrm{m}, 220 \times 4.6 \mathrm{~mm}$; Brownlee Applied Biosystem, CA, USA) as previously described by Hallström et al. ${ }^{14}$ The mobile phase consisted of $2 \mathrm{mM} \mathrm{H}_{2} \mathrm{SO}_{4}$ and the flow rate was $0.3 \mathrm{ml} / \mathrm{min}$. An UV detector at $214 \mathrm{~nm}$ was used for the peak detection.

Glutamate was analyzed by HPLC with fluorescence detection after precolumn o-phthaldialdehyde (OPA) derivatization. ${ }^{15}$ The method was modified to suit the microdialysis samples, with a Nucleosil $\mathrm{C}_{18}$ reverse phase column $(5 \mu \mathrm{m}: 60 \times 4 \mathrm{~mm}$; Knauer, Germany) eluted with a buffer consisting of $0.1 \mathrm{M}$ sodium acetate $(\mathrm{pH} 6.95), 5 \%(\mathrm{v} / \mathrm{v})$ methanol and $2.5 \%(\mathrm{v} / \mathrm{v})$ tetrahydrofuran. The flow rate was $1.2 \mathrm{ml} /$ min. Ten microliters of the sample was mixed with $10 \mu \mathrm{l}$ of OPA reagent by a CMA 200 autoinjector. The amino acid-OPA complex was detected by a Hitachi F 1000 fluroescence detector with an excitation wavelength of $330 \mathrm{~nm}$ and an emission wavelength of $440 \mathrm{~nm}$ or by a CMA 280 fluorescence detector with a fixed wavelength (a tungsten lamp, excitation $340-360 \mathrm{~nm}$ ) and a filter (emission maximum $495 \mathrm{~nm}$ ).

Glucose was analyzed on a CMA-600 (CMA Microdialysis $\mathrm{AB}$ ) analyser. The method used was an oxidation of glucose by glucose oxidase (GOD) which forms gluconic acid and $\mathrm{H}_{2} \mathrm{O}_{2}$. Peroxidase (POD) then catalysed the reaction between the hydrogen peroxide formed and phenol and 4-aminoantipyrine to form the coloured substance quinonemine. The rate of formation of the coloured substance was then measured photometrically at $546 \mathrm{~nm} .^{16}$

\section{Statistical analysis}

Data are given as median after calculation of the mean value of the measurements during the last $30 \mathrm{~min}$ before clamping, and then transformation of this value into $100 \%$. Changes were then calculated and presented in percentage of this basal value. Differences between samplings were assessed by two-tailed Wilcoxon signed ranks test with a probability less than 0.5 considered as significant.

Ethics

The experiments were performed from September 1995 to January 1997 and the protocol was approved by the local responsible laboratory animal science specialist 
under the surveillance of the Norwegian Animal Research Authority (NARA) and registered by the Authority.

\section{Results}

Glucose levels were analyzed inconsistently due to small amounts of microdialysate, but decreased $40 \%$ or more during cross-clamping. Table 1 shows the median and range of pre-clamping values of glutamate, lactate,

Table 1 Basal microdialysis levels during $10 \mathrm{~min}$ stabilisation prior to aortic clamping. Values as median and range

\begin{tabular}{lcc}
\hline Substance & \multicolumn{2}{c}{ Median (range) } \\
\hline Glutamate & 3.2 & $(0.8-13.5) \mu \mathrm{M}$ \\
Lactate & 230.3 & $(129.0-996.0) \mathrm{mM}$ \\
Pyruvate & 38.1 & $(15.4-70.9) \mu \mathrm{M}$ \\
Lactate/pyruvate & 6.54 & $(3.87-18.3)$ \\
\hline
\end{tabular}

A

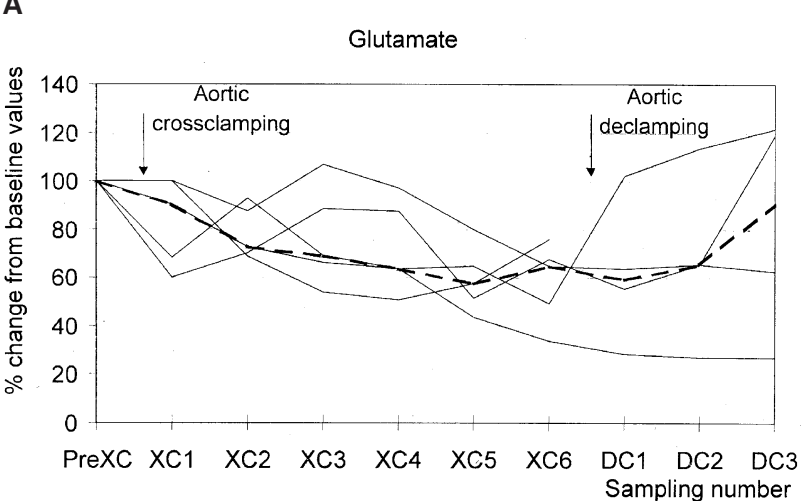

C

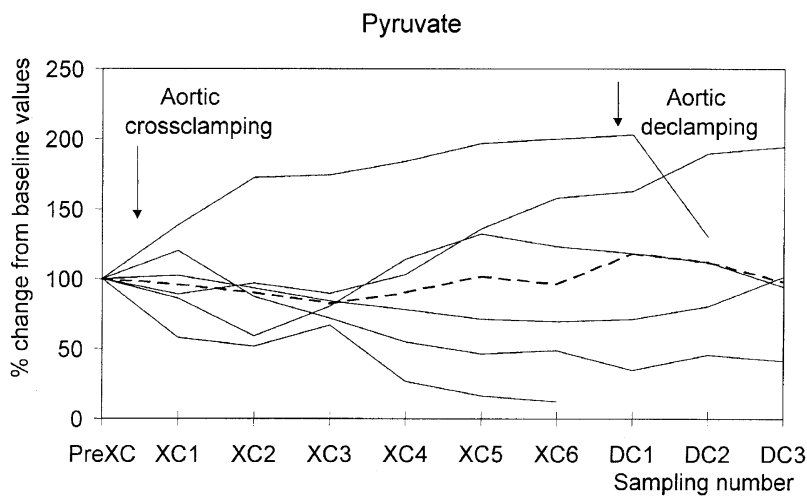

pyruvate and the lactate-pyruvate ratio. Initially, lactate levels increased and pyruvate levels decreased slightly, followed by an insignificant increase during the clamping period. The lactate-pyruvate ratio increased correspondingly, with the last three measurements before declamping representing a significant increase $(P=0.031)$ from preclamping values. Figure $1 \mathrm{~B}-\mathrm{D}$ depict the course of the metabolite concentrations. At $30 \mathrm{~min}$ of ischaemia, the median lactate-pyruvate ratio had increased by $128 \%$, and at $60 \mathrm{~min}$ by $169 \%$. There was no evident change of this ratio between the ischaemic and the reperfusion period, but lactate levels increased slightly more during reperfusion.

It was only possible to analyze glutamate in five out of six pigs, due to technical problems. There was a tendency that glutamate levels decreased from baseline levels during the cross-clamp period, but this decrease did not reach the level of significance $(P=0.063)$. During the last $10 \mathrm{~min}$ sampling of the $30 \mathrm{~min}$ reperfusion period, there was a tendency to increases
B

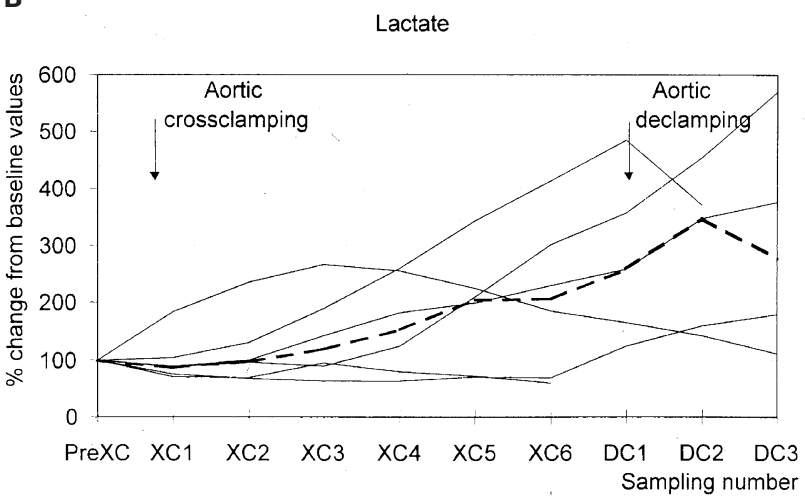

D

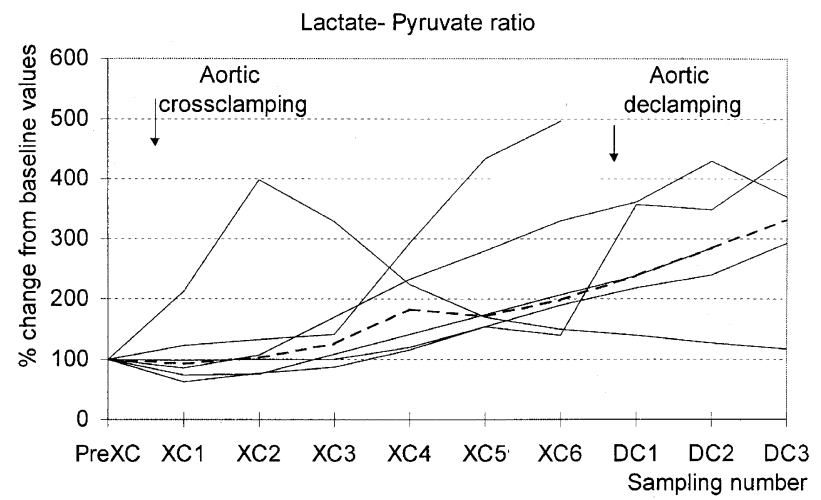

Figure 1 (A) Glutamate levels as per cent change from basal values. It was only possible to analyze glutamate in five out of six pigs, due to technical problems. (B) Lactate levels as per cent change from basal values. (C) Pyruvate levels as per cent change from basal values. (D) Lactate-pyruvate ratio values as per cent change from basal levels. (A-D) all have the same format and depict the time-course of the measurements. Per cent change from basal values is shown on the ordinate, with the individual basal values all recalculated to 100. PreXC: basal value before cross-clamping: XC1-XC6 and DC1-DC3: samples drawn every $10 \mathrm{~min}$ during $1 \mathrm{~h}$ of aortic cross-clamping (XC) and $30 \mathrm{~min}$ after declamping (DC) respectively. The thin, continuous line connects the individual measurements for each animal and the thicker, broken line represents the median value. There are missing values in the reperfusion period for one animal in all measurements, due to technical reasons 


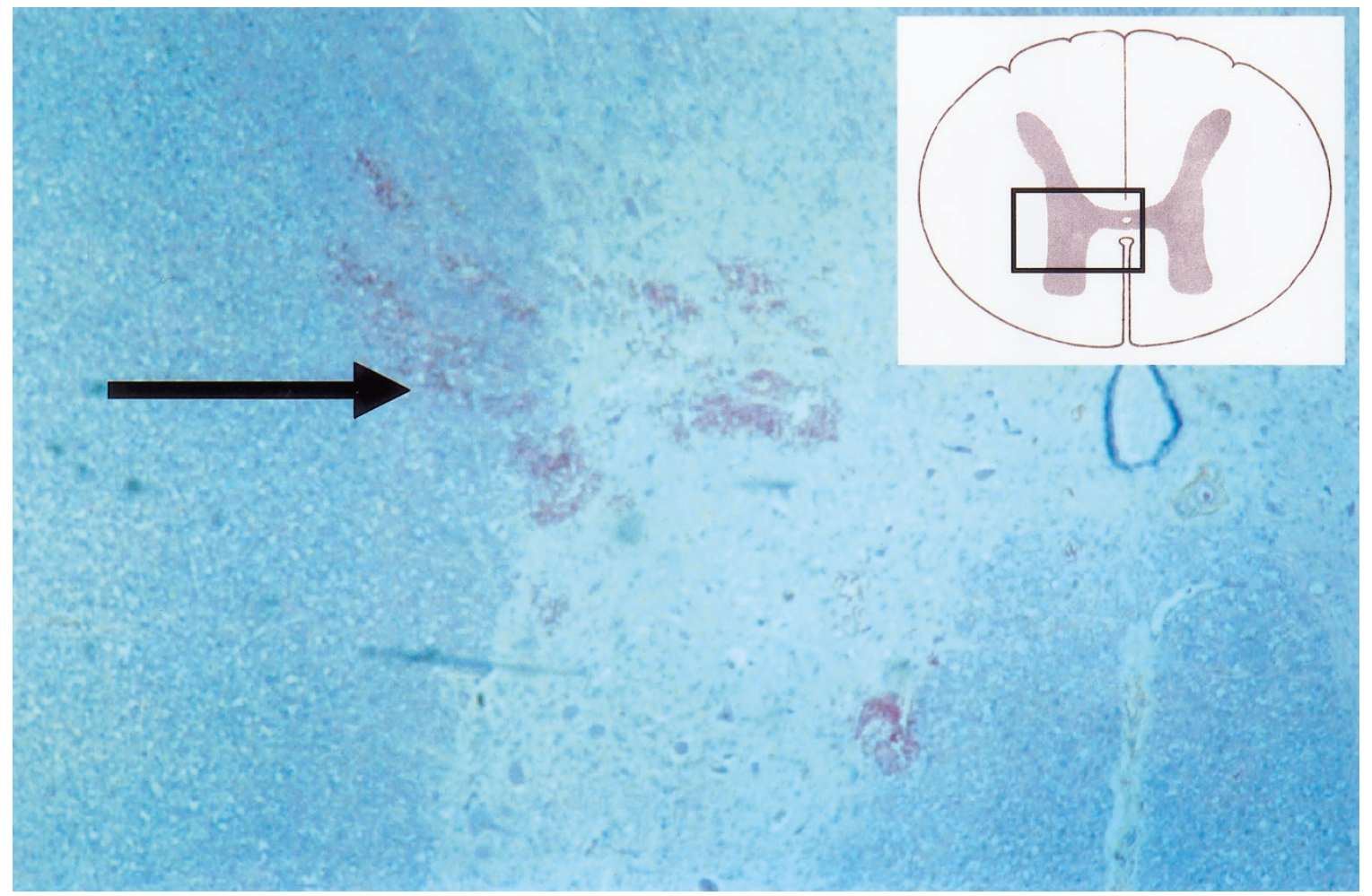

Figure 2 Histopathology of the spinal cord. The quadrant in the right corner represents the area depicted. The darker blue area is white matter, and the brighter area grey matter. The arrow is pointing at traces of bleeding along the microdialysis probe channel in grey and white matter

in glutamate levels not seen during the cross-clamp period.

\section{Discussion}

In this series, aortic cross-clamping induced significant increase of the lactate/pyruvate ratio, corresponding to findings expected in muscle ischaemia. We failed, however, to show significant increases of glutamate levels, contrary to the results from Rokkas et al, ${ }^{12,13}$ who also showed that pre-treatment with dextrorphane ${ }^{12}$ and hypothermia ${ }^{13}$ prevented glutamate increases.

Without knowledge of all details to compare the experimental settings between Rokkas' studies and the present experiments, it is difficult to explain these differences in results. Both studies have utilised laminectomy to facilitate the insertion of the microdialysis probes, and it has previously been shown that laminectomy may cause a decline of the spinal cord blood flow (SCBF). In a feline model with one segment laminectomy and intact dura mater, Anderson et al, ${ }^{17}$ utilising radio-labelled microspheres, found a significantly $(22 \%$ to $45 \%)$ reduced SCBF along the entire length of the spinal cord at $15 \mathrm{~min}$ after the laminectomy. With the laminectomy site closed, SCBF approached pre-laminectomy levels $1 \mathrm{~h}$ after closure, but there were still areas of reduced flow along the spinal cord. The authors speculated that a tempera- ture-induced vasoconstriction could explain these findings.

It is less probable that a lowered blood flow to the spinal cord, locally or generally, would be the explanation for the lack of increased glutamate levels, due to the fact that the increase in glutamate levels per se should depend on the reduced blood flow during the aortic cross clamp period. On the other hand, it cannot be excluded that a reduced temperature of the spinal cord, caused by exposure of the spinal canal could act as a 'hypothermic' condition, able to prevent glutamatae release. Another explanation might be that $60 \mathrm{~min}$ of aortic cross-clamping is insufficient to allow for glutamate to be released, or that ischaemia was not complete. From our previous experiments, measuring microcirculation with laserDoppler technique, it was evident that the crossclamping procedure including occlusion of the azygos vein produces a significant decrease of tissue perfusion, even if there is some collateral circulation left. In those studies the laser Doppler probe was inserted percutaneously making only a small puncture of the dura, and therefore spinal fluid pressure could be maintained and contribute to a reduced perfusion of the spinal cord.

A further possibility for the lack of glutamate increase could be that the microdialysis probe was incorrectly inserted into the grey matter. In two cases histologic studies were performed, showing convin- 
cingly however, that the probe was located in the grey matter (Figure 2).

In our experiments no samples were taken during the initial $60 \mathrm{~min}$ stabilisation period, but the first samples taken just before clamping showed higher glutamate levels than what was achieved during the aortic cross clamp period. The most probable explanation for this initial increase in glutamate levels is a glutamate release immediately after insertion of the probe as a response to the trauma. This finding also gives evidence that the probe was localised in an anatomical area where glutamate should be found, if released. The tendency to increases of glutamate levels during the last $10 \mathrm{~min}$ of sampling during the $30 \mathrm{~min}$ reperfusion period, points in the same direction.

Our main findings are thus changes of lactate and pyruvate levels, corresponding to muscle ischaemia. This result raises the question whether substances like allopurinol, known to reduce reperfusion injury of muscle, could be of value to reduce ischaemic damage of the spinal cord.

Our experimental setting does not enable correlation to any clinical findings of paraplegia, but other series using pigs, in which neurologic status was examined after the animals had returned to consciousness after $30 \mathrm{~min}$ of aortic cross-clamping ${ }^{18}$ revealed that nontreated animals were all paraplegic, while those treated with a combination of allopurinol and deferoxamine all recovered totally. This protective effect was not explained by any improved spinal cord blood flow during the cross-clamping period in the pre-treated animals.

Since paraplegia following thoracic and thoracoabdominal aortic surgery still is a major problem, we think it is important to study further the metabolic response of the spinal cord during proximal aortic cross clamping. Microdialysis seems to give good opportunities to study such phenomena, but further questions have to be addressed, such as factors which possibly reduce the chance of glutamate increase during ischaemia, perfusion conditions of the spinal cord in our experimental setting, using laser Doppler flux measurements or colour-labelled microspheres. Also direct measurements of oxygen tension in the cord might be helpful to estimate the level of ischaemia. This kind of experiment is presently going on.

\section{Acknowledgements}

The authors wish to thank Tonje Strømholm MD.PhD. Department of Pathology, Trondheim University Hospital, for the histopathological studies and the preparation of Figure 2.

\section{References}

1 Shenaq SA, Svensson LG. Paraplegia following aortic surgery. $J$ Cardiothorac Vasc Anesth 1993; 7: 81 - 94.

2 Myhre HO, Saether OD, Stenseth R, Aadahl P. Attempts to reduce paraplegia risk in aortic surgery. In: Greenhalgh RM, Mannick JA (eds). The cause and management of aneurysms. W.B. Saunders: London 1990, pp 373-385.

3 Aadahl P, Saether OD, Stenseth R, Myhre HO. Microcirculation of the spinal cord during proximal aortic cross-clamping. Eur $J$ Vasc Surg 1990; 4: 5-10.

4 Ungerstedt U. Microdialysis - principles and applications for studies in animals and man. $J$ Int Med 1991; 230: 365-373.

5 Ungerstedt U. Microdialysis - a new technique for monitoring local tissue events in the clinic. Acta Anaesthesiol Scand Suppl 1997; 110: 123

6 Hamberger A, Jacobson I, Nyström B, Sandberg M. Microdialysis sampling of neuronal environment in basic and clinical research. J Int Med 1991; 230: 375 - 380 .

7 Benveniste H, Drejer J, Schousboe A, Diemer NH. Elevation of the extracellular concentrations of glutamate and aspartate in rat hippocampus during transient cerebral ischemia monitored by intracerebral microdialysis. J Neurochem 1984; 43: 1369- 1374.

8 Rothman SM, Olney JW. Glutamate and the pathophysiology of hypoxic-ischemic brain damage. Ann Neurol 1986; 19: 105-111.

9 Farooque M, Hillered L, Holtz A, Olsson Y. Changes of extracellular levels of amino acids after graded compression trauma to the spinal cord: An experimental study in the rat using microdialysis. J Neurotrauma 1996; 13: $537-548$.

10 Simpson Jr RK, Robertson CS, Goodman JC. Spinal cord ischemia-induced elevation of amino-acids: Extracellular measurement with microdialysis. Neurochem Res 1990; 15: 635-639.

11 Choi DW, Rothman SM. The role of glutamate neurotoxicity in hypoxic-ischemic neuronal death. Ann Rev Neurosci 1990; 13: $171-182$.

12 Rokkas CK et al. Dextrorphan inhibits the release of excitatory amino acids during spinal cord ischemia. Ann Thorac Surg 1994; 58: $312-319$

13 Rokkas et al. Profound systemic hypothermia inhibits the release of neurotransmitter amino acids in spinal cord ischemia. $J$ Thorac Cardiovasc Surg 1995; 110: 27 - 35.

14 Hallstrøm Å, Carlsson A, Hillered L, Ungerstedt U. Simultaneous determination of lactate, pyruvate, and ascorbate in microdialysis samples from rat brain, blood, fat and muscle using high performance liquid chromatography. J Pharmacol Methods 1989; 21: $113-1124$.

15 Lindroth P, Mopper K. High performance liquid chromatographic determination of subpicomole amounts of amino acids by precolumn fluorescence derivatization with o-phthaldialdehyde. Anal Chem 1979; 51: 16667-16674.

16 Barham D, Trinder P. An improved colour reagent for the determination of blood glucose by the oxidase system. Analyst 1972; 97: $142-145$.

17 Anderson DK, Nicolosi GR, Means ED, Hartley LE. Effects of laminectomy on spinal cord flow. J Neurosurg 1978; 48: 232-238.

18 Quaymi A et al. Additive effect of allopurinol and deferoxamine in the prevention of spinal cord injury caused by aortic crossclamping. J Thorac Cardiovasc Surg 1994; 107: 1203 - 1209. 